\title{
SEARCH FOR SOURCES OF HIGH-ENERGY NEUTRONS WITH FOUR YEARS OF DATA FROM THE ICETOP DETECTOR
}

M. G. Aartsen ${ }^{1}$, K. Abraham ${ }^{2}$, M. Ackermann ${ }^{3}$, J. Adams ${ }^{4}$, J. A. Aguilar ${ }^{5}$, M. Ahlers ${ }^{6}$, M. Ahrens ${ }^{7}$, D. Altmann ${ }^{8}$, K. Andeenn ${ }^{9}$, T. Anderson ${ }^{10}$, I. Ansseau ${ }^{5}$, G. Anton ${ }^{8}$, M. Archinger $^{11}$, C. Argüelles ${ }^{12}$, J. Auffenberg $^{13}$, S. Axani $^{12}$, X. Bai ${ }^{14}, \mathrm{~S}$. W. Barwick ${ }^{15}$, V. Baum ${ }^{11}$, R. BaY ${ }^{16}$, J. J. BeattY ${ }^{17,18}$, J. Becker Tuus ${ }^{19}$, K.-H. Becker ${ }^{20}$, S. BenZvi ${ }^{21}$ P. Berghaus ${ }^{22}$, D. Berley ${ }^{23}$, E. Bernardini ${ }^{3}$, A. Bernhard ${ }^{2}$, D. Z. Besson ${ }^{24}$, G. Binder ${ }^{16,25}$, D. Bindig ${ }^{20}$, M. BissoK ${ }^{13}$, E. Blaufuss ${ }^{23}$, S. Blot ${ }^{3}$, C. Bohm ${ }^{7}$, M. Börner ${ }^{26}$, F. Bos ${ }^{19}$, D. Bose ${ }^{27}$, S. Böser ${ }^{11}$, O. Botner ${ }^{28}$, J. Braun ${ }^{6}$, L. BraYeur ${ }^{29}$, H.-P. Bretz ${ }^{3}$, A. Burgman ${ }^{28}$, T. Carver ${ }^{30}$, M. Casier ${ }^{29}$, E. Cheung ${ }^{23}$, D. Chirkin ${ }^{6}$, A. Christov ${ }^{30}$, K. Clark ${ }^{31}$, L. Classen $^{32}$, S. Coenders' ${ }^{2}$, G. H. Collin ${ }^{12}$, J. M. Conrad ${ }^{12}$, D. F. Cowen ${ }^{10,33}$, R. Cross ${ }^{21}$, M. DAY ${ }^{6}$, J. P. A. M. DE ANDrÉ ${ }^{34}$,

C. De Clerce ${ }^{29}$, E. del Pino Rosendo ${ }^{11}$, H. Dembinski ${ }^{35}$, S. De Ridder ${ }^{36}$, P. Desiati ${ }^{6}$, K. D. De Vries ${ }^{29}$, G. De Wasseige ${ }^{29}$, M. DE With ${ }^{37}$, T. DeYoung ${ }^{34}$, J. C. Díaz-VÉlez ${ }^{6}$, V. Di Lorenzo ${ }^{11}$, H. Dujmovic ${ }^{27}$, J. P. Dumm ${ }^{7}$, M. DunKman ${ }^{10}$,

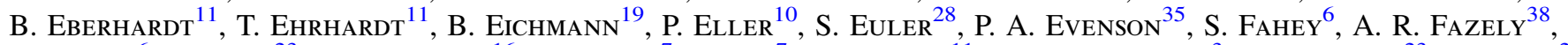
J. Feintzeig ${ }^{6}$, J. Felde ${ }^{23}$, K. Filimonov ${ }^{16}$, C. Finley ${ }^{7}$, S. Flis ${ }^{7}$, C.-C. Fösig ${ }^{11}$, A. Franckowiak ${ }^{3}$, E. Friedman ${ }^{23}$, T. FuChs ${ }^{26}$, T. K. Gaisser ${ }^{35}$, J. Gallagher ${ }^{39}$, L. Gerhardt ${ }^{16,25}$, K. Ghorbani ${ }^{6}$, W. Giang ${ }^{40}$, L. Gladstone ${ }^{6}$, M. Glagla ${ }^{13}$, T. Glüsenkamp ${ }^{3}$, A. Goldschmidt ${ }^{25}$, G. Golup ${ }^{29}$, J. G. GonZAlez ${ }^{35}$, D. Grant ${ }^{40}$, Z. Griffith ${ }^{6}$, C. HaAcK ${ }^{13}$, A. Haj Ismail $^{36}$,

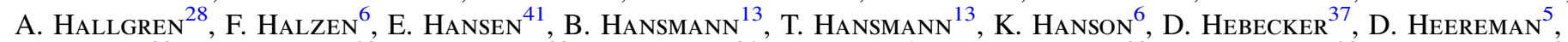

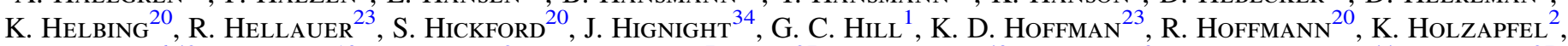

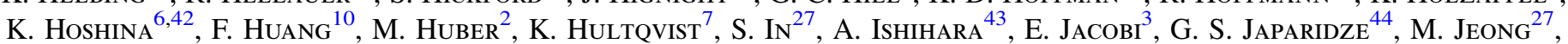

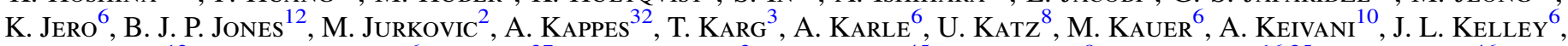

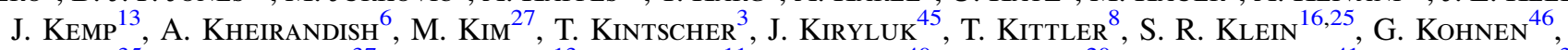
R. Koirala ${ }^{35}$, H. Kolanoski ${ }^{37}$, R. Konietz ${ }^{13}$, L. KöPKe ${ }^{11}$, C. Kopper ${ }^{40}$, S. Kopper ${ }^{20}$, D. J. Koskinen ${ }^{41}$, M. KowalsKi ${ }^{37,3}$, K. Krings 2 , M. Kroll ${ }^{19}$, G. KrüCKL ${ }^{11}$, C. KrüGer ${ }^{6}$, J. Kunnen ${ }^{29}$, S. Kunwar ${ }^{3}$, N. Kurahashi ${ }^{47}$, T. Kuwabara ${ }^{43}$, M. Labare $^{36}$, J. L. Lanfranchi ${ }^{10}$, M. J. Larson ${ }^{41}$, F. Lauber ${ }^{20}$, D. LenNarz ${ }^{34}$, M. LesiaK-BzdaK ${ }^{45}$, M. LeuermanN ${ }^{13}$,

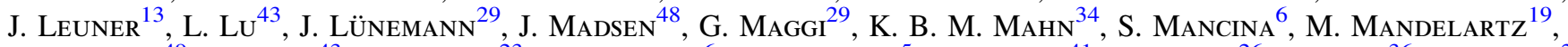
R. Maruyama ${ }^{49}$, K. Mase ${ }^{43}$, R. Maunu ${ }^{23}$, F. McNally ${ }^{6}$, K. Meagher ${ }^{5}$, M. Medici ${ }^{41}$, M. Meier ${ }^{26}$, A. Meli ${ }^{36}$, T. Menne ${ }^{26}$,

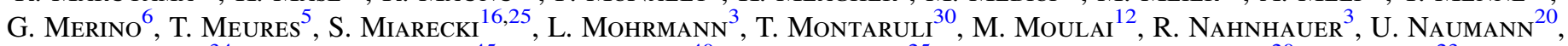
G. NeER $^{34}$, H. Niederhausen ${ }^{45}$, S. C. Nowicki ${ }^{40}$, D. R. Nygren ${ }^{25}$, A. Obertacke Pollmann ${ }^{20}$, A. Olivas ${ }^{23}$,

A. O’Murchadha ${ }^{5}$, T. Palczewski ${ }^{50}$, H. Pandya ${ }^{35}$, D. V. Pankova ${ }^{10}$, Ö. Pener ${ }^{13}$, J. A. Pepper ${ }^{50}$, C. Pérez de los Heros ${ }^{28}$,

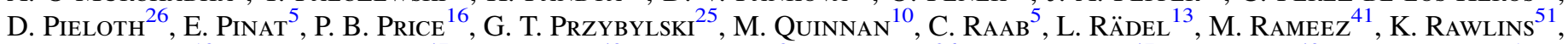

R. Reimann ${ }^{13}$, B. Relethford ${ }^{47}$, M. Relich ${ }^{43}$, E. Resconi ${ }^{2}$, W. Rhode ${ }^{26}$, M. Richman ${ }^{47}$, B. Riedel ${ }^{40}$, S. Robertson ${ }^{1}$,

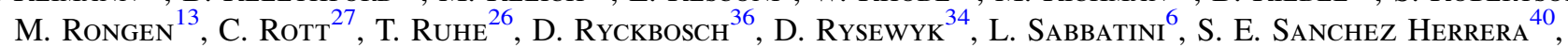

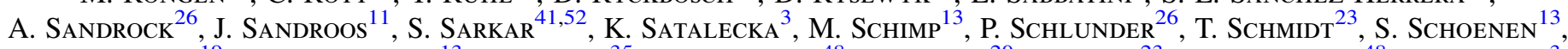
S. Schöneberg ${ }^{19}$, L. Schumacher ${ }^{13}$, D. Seckel ${ }^{35}$, S. Seunarine ${ }^{48}$, D. Soldin ${ }^{20}$, M. Song ${ }^{23}$, G. M. SpiczaK ${ }^{48}$, C. Spiering ${ }^{3}$, M. Stahlberg ${ }^{13}$, T. Stanev ${ }^{35}$, A. Stasik ${ }^{3}$, A. Steuer ${ }^{11}$, T. Stezelberger ${ }^{25}$, R. G. Stokstad ${ }^{25}$, A. Stössl ${ }^{3}$, R. Ström ${ }^{28}$,

N. L. Strotjohann ${ }^{3}$, G. W. Sullivan ${ }^{23}$, M. Sutherland ${ }^{17}$, H. Taavola ${ }^{28}$, I. Taboada ${ }^{53}$, J. Tatar ${ }^{16,25}$, F. Tenholt ${ }^{19}$,

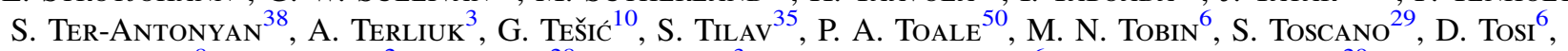

M. Tselengidou ${ }^{8}$, A. Turcati ${ }^{2}$, E. Unger ${ }^{28}$, M. Usner $^{3}$, J. VAndenbroucke ${ }^{6}$, N. van Eijndhoven ${ }^{29}$, S. Vanheule $^{36}$, M. van Rossem ${ }^{6}$, J. van Santen ${ }^{3}$, J. Veenkamp ${ }^{2}$, M. Vehring ${ }^{13}$, M. Voge $^{54}$, M. Vraeghe ${ }^{36}$, C. Walck ${ }^{7}$, A. Wallace ${ }^{1}$, M. WallrafF ${ }^{13}$, N. Wandkowsky ${ }^{6}$, Ch. Weaver ${ }^{40}$, M. J. Weiss ${ }^{10}$, C. Wendt $^{6}$, S. Westerhoff ${ }^{6}$, B. J. Whelan ${ }^{1}$, S. WickmanN ${ }^{13}$, K. Wiebe ${ }^{11}$, C. H. Wiebusch ${ }^{13}$, L. Wille ${ }^{6}$, D. R. Williams ${ }^{50}$, L. Wills ${ }^{47}$, M. Wolf ${ }^{7}$, T. R. Wood ${ }^{40}$,

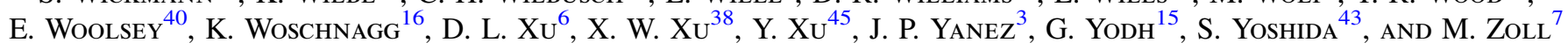

(IceCube Collaboration)

${ }^{1}$ Department of Physics, University of Adelaide, Adelaide, 5005, Australia

${ }^{2}$ Physik-department, Technische Universität München, D-85748 Garching, Germany ${ }^{3}$ DESY, D-15735 Zeuthen, Germany

${ }^{4}$ Department of Physics and Astronomy, University of Canterbury, Private Bag 4800, Christchurch, New Zealand

${ }^{5}$ Université Libre de Bruxelles, Science Faculty CP230, B-1050 Brussels, Belgium

${ }^{6}$ Department of Physics and Wisconsin IceCube Particle Astrophysics Center, University of Wisconsin, Madison, WI 53706, USA

${ }^{7}$ Oskar Klein Centre and Department of Physics, Stockholm University, SE-10691 Stockholm, Sweden

${ }^{8}$ Erlangen Centre for Astroparticle Physics, Friedrich-Alexander-Universität Erlangen-Nürnberg, D-91058 Erlangen, Germany 9 Department of Physics, Marquette University, Milwaukee, WI 53201, USA

${ }^{10}$ Department of Physics, Pennsylvania State University, University Park, PA 16802, USA

${ }^{11}$ Institute of Physics, University of Mainz, Staudinger Weg 7, D-55099 Mainz, Germany

${ }^{12}$ Department of Physics, Massachusetts Institute of Technology, Cambridge, MA 02139, USA

${ }^{14}$ III. Physikalisches Institut, RWTH Aachen University, D-52056 Aachen, Germany
Physics Department, South Dakota School of Mines and Technology, Rapid City, SD 57701, USA

${ }^{15}$ Department of Physics and Astronomy, University of California, Irvine, CA 92697, USA

${ }^{17}$ Department of Physics and Center for Cosmology and Astro-Particle Physics, Ohio State University, Columbus, OH 43210, USA 


\author{
${ }^{18}$ Department of Astronomy, Ohio State University, Columbus, OH 43210, USA \\ ${ }^{19}$ Fakultät für Physik \& Astronomie, Ruhr-Universität Bochum, D-44780 Bochum, Germany \\ ${ }^{20}$ Department of Physics, University of Wuppertal, D-42119 Wuppertal, Germany \\ ${ }^{21}$ Department of Physics and Astronomy, University of Rochester, Rochester, NY 14627, USA \\ 22 National Research Nuclear University MEPhI (Moscow Engineering Physics Institute), Moscow, Russia \\ ${ }^{23}$ Department of Physics, University of Maryland, College Park, MD 20742, USA \\ ${ }^{24}$ Department of Physics and Astronomy, University of Kansas, Lawrence, KS 66045, USA \\ ${ }^{25}$ Lawrence Berkeley National Laboratory, Berkeley, CA 94720, USA \\ 26 Department of Physics, TU Dortmund University, D-44221 Dortmund, Germany \\ 27 Department of Physics, Sungkyunkwan University, Suwon 440-746, Korea \\ 28 Department of Physics and Astronomy, Uppsala University, Box 516, S-75120 Uppsala, Sweden \\ ${ }^{29}$ Vrije Universiteit Brussel, Dienst ELEM, B-1050 Brussels, Belgium \\ ${ }^{30}$ Département de physique nucléaire et corpusculaire, Université de Genève, CH-1211 Genève, Switzerland \\ ${ }^{31}$ Department of Physics, University of Toronto, Toronto, ON M5S 1A7, Canada \\ 32 Institut für Kernphysik, Westfälische Wilhelms-Universität Münster, D-48149 Münster, Germany \\ 33 Department of Astronomy and Astrophysics, Pennsylvania State University, University Park, PA 16802, USA \\ ${ }^{34}$ Department of Physics and Astronomy, Michigan State University, East Lansing, MI 48824, USA \\ ${ }^{35}$ Bartol Research Institute and Department of Physics and Astronomy, University of Delaware, Newark, DE 19716, USA \\ ${ }^{36}$ Department of Physics and Astronomy, University of Gent, B-9000 Gent, Belgium \\ 37 Institut für Physik, Humboldt-Universität zu Berlin, D-12489 Berlin, Germany \\ ${ }^{38}$ Department of Physics, Southern University, Baton Rouge, LA 70813, USA \\ 39 Department of Astronomy, University of Wisconsin, Madison, WI 53706, USA \\ ${ }_{41}^{40}$ Department of Physics, University of Alberta, Edmonton, AB T6G 2E1, Canada \\ ${ }^{41}$ Niels Bohr Institute, University of Copenhagen, DK-2100 Copenhagen, Denmark \\ 42 Earthquake Research Institute, University of Tokyo, Bunkyo, Tokyo 113-0032, Japan \\ ${ }^{43}$ Department of Physics, Chiba University, Chiba 263-8522, Japan \\ 44 CTSPS, Clark-Atlanta University, Atlanta, GA 30314, USA \\ 45 Department of Physics and Astronomy, Stony Brook University, Stony Brook, NY 11794-3800, USA \\ ${ }^{46}$ Université de Mons, B-7000 Mons, Belgium \\ ${ }^{47}$ Department of Physics, Drexel University, 3141 Chestnut Street, Philadelphia, PA 19104, USA \\ ${ }^{48}$ Department of Physics, University of Wisconsin, River Falls, WI 54022, USA \\ ${ }^{50}$ Department of Physics, Yale University, New Haven, CT 06520, USA
Department of Physics and Astronomy, University of Alabama, Tuscaloosa, AL 35487, USA \\ 51 Department of Physics and Astronomy, University of Alaska Anchorage, 3211 Providence Drive, Anchorage, AK 99508, USA \\ 52 Department of Physics, University of Oxford, 1 Keble Road, Oxford OX1 3NP, UK \\ ${ }^{53}$ School of Physics and Center for Relativistic Astrophysics, Georgia Institute of Technology, Atlanta, GA 30332, USA \\ 54 Physikalisches Institut, Universität Bonn, Nussallee 12, D-53115 Bonn, Germany \\ Received 2016 July 19; revised 2016 August 9; accepted 2016 August 9; published 2016 October 18
}

\title{
ABSTRACT
}

IceTop is an air-shower array located on the Antarctic ice sheet at the geographic South Pole. IceTop can detect an astrophysical flux of neutrons from Galactic sources as an excess of cosmic-ray air showers arriving from the source direction. Neutrons are undeflected by the Galactic magnetic field and can typically travel $10(E / \mathrm{PeV}) \mathrm{pc}$ before decay. Two searches are performed using $4 \mathrm{yr}$ of the IceTop data set to look for a statistically significant excess of events with energies above $10 \mathrm{PeV}\left(10^{16} \mathrm{eV}\right)$ arriving within a small solid angle. The all-sky search method covers from $-90^{\circ}$ to approximately $-50^{\circ}$ in declination. No significant excess is found. A targeted search is also performed, looking for significant correlation with candidate sources in different target sets. This search uses a higher-energy cut $(100 \mathrm{PeV})$ since most target objects lie beyond $1 \mathrm{kpc}$. The target sets include pulsars with confirmed $\mathrm{TeV}$ energy photon fluxes and high-mass X-ray binaries. No significant correlation is found for any target set. Flux upper limits are determined for both searches, which can constrain Galactic neutron sources and production scenarios.

Key words: astroparticle physics - cosmic rays - methods: data analysis

\section{INTRODUCTION}

The Galactic magnetic field (GMF) strongly affects the arrival distribution of charged cosmic rays, thereby obscuring their sources. A compact source of high-energy neutrons would manifest as a point source in cosmic-ray arrival directions since neutrons are not deflected by magnetic fields. Secondary neutral particles are an expected signature of hadronic acceleration in Galactic sources. Neutral particles would be produced as the cosmic-ray protons and nuclei undergo $p p$ and $p \gamma$ collisions and photodisintegration, respectively, on the ambient photons and cosmic rays within the dense environment surrounding their source (see, e.g., Candia et al. 2002; Crocker et al. 2005; Cavasinni et al. 2006; Anchordoqui et al. 2007). For example, neutrons result from charge-exchange interactions,

$$
p \gamma \rightarrow n \pi^{+}
$$

where a $\pi^{+}$emerges with the proton's positive charge and the neutron retains most of the energy. For interacting proton primaries, photons resulting from $\pi^{0}$ decays take a small fraction of the proton energy. The production of neutrons exceeds the production of photons at the same energy (Crocker et al. 2005).

It is plausible that known Galactic sources could produce high-energy neutron fluxes, based on the measured TeV energy photon flux. For some Galactic sources, the energy flux of TeV photons is greater than $1 \mathrm{eV} \mathrm{cm}^{-2} \mathrm{~s}^{-1}$ (Hinton \& Hofmann 2009). Sources producing particle fluxes with an $E^{-2}$ 
differential energy spectrum inject equal energy into each energy decade. If sources in the Galaxy produce PeV photons in addition to $\mathrm{TeV}$ photons, the $\mathrm{PeV}$ photon energy flux would also exceed $1 \mathrm{eV} \mathrm{cm}^{-2} \mathrm{~s}^{-1}$ at Earth. For sources that produce neutrons by hadronic processes as well, the neutron energy flux would be even higher since the neutron production rate exceeds the photon production rate, as noted previously.

Free neutrons undergo beta decay with a $880.0 \pm 0.9 \mathrm{~s}$ halflife (Olive et al. 2014). Due to this decay, sources will only be visible within about $10(E / \mathrm{PeV})$ pc of Earth. Since plausible accelerators such as young pulsars are no closer than $100 \mathrm{pc}$, searches at energies above $10 \mathrm{PeV}$ are the most promising.

A diffuse flux of neutrons could be expected from interactions of cosmic-ray primaries with ambient photons and the interstellar medium. However, at PeV energies this flux would appear all over the sky since the effective range is less than the thickness of the Galactic disk. This complicates a search for correlations with the Galactic plane since an excess signal could not be constrained to a particular region of the sky, for example, Galactic latitudes $|b|<10^{\circ}$.

At energies above $10^{18} \mathrm{eV}(1 \mathrm{EeV})$, the Pierre Auger Observatory recently performed a search for neutrons in the southern hemisphere, finding no significant signal excesses or correlations with catalogs of Galactic objects, and established flux upper limits (Aab et al. 2012, 2014). The Telescope Array experiment has established flux limits for point sources above $0.5 \mathrm{EeV}$ in the northern hemisphere (Abbasi et al. 2015). KASCADE (Antoni et al. 2004) and CASA-MIA (Chantell et al. 1997; Borione et al. 1998) found no point sources in the northern hemisphere, also setting flux limits (an all-sky limit in the case of KASCADE). AGASA (Hayashida et al. 1999) and a reanalysis (Bellido et al. 2001) of SUGAR data reported slight excesses toward the Galactic center, although these were later not confirmed by Auger (Aab et al. 2015).

This paper reports the results of two searches for point-like signals in the arrival direction distribution of $4 \mathrm{yr}$ of IceTop data. The two searches are an all-sky search for general hot spots on the sky and a search for correlations with nearby known Galactic sources. In the all-sky search, we look for an excess of events from any direction in the sky, evaluating the significance of any excess using the method of Li \& Ma (1983). The observable signature of a neutron flux is an excess of proton-like air showers. The targeted search is treated as a stacked analysis using a set of candidate sources from an astrophysical catalog. It is assumed that many or all of the candidates for a given set are emitting neutrons, so the combined signal should be more significant than that of a single target. In both the all-sky and targeted searches, we set flux upper limits using the procedures of Feldman \& Cousins (1998).

This paper is organized as follows. In Section 2, the IceTop detector is described. Section 3 summarizes the reconstruction methods and characteristics of the data set. The analysis methods and details of the search methods are described in Section 4. The search results are presented in Section 5. A discussion of the results (Section 6) concludes the paper.

\section{ICECUBE/ICETOP}

IceTop is the surface air-shower array of the IceCube Neutrino Observatory at the geographical South Pole located $2835 \mathrm{~m}$ above sea level (Abbasi et al. 2013). Its final configuration consists of 81 stations covering $1 \mathrm{~km}^{2}$ with an
Table 1

Detector Configurations and Their Respective Number of Events and Effective Snow Attenuation Lengths for All Years Used in This Analysis

\begin{tabular}{lccc}
\hline \hline Configuration & $\begin{array}{c}\text { Live Time } \\
\text { (days) }\end{array}$ & $\begin{array}{c}\text { Number of Events } \\
N_{>10 \mathrm{PeV}}\left(N_{>100 \mathrm{PeV}}\right)\end{array}$ & $\begin{array}{c}\text { Snow Depth } \\
(\mathrm{m})\end{array}$ \\
\hline IC79 & 327.3 & $291,738(2986)$ & 2.1 \\
IC86-1 & 342.0 & $305,138(3173)$ & 2.25 \\
IC86-2 & 332.3 & $306,868(3025)$ & 2.25 \\
IC86-3 & 362.2 & $329,743(3374)$ & 2.3 \\
\hline Total & 1368.8 & $1,233,487(12,558)$ & $\ldots$ \\
\hline
\end{tabular}

average station separation of $125 \mathrm{~m}$. Detector construction started in 2005 and finished in 2010. A single station consists of two light-tight tanks separated by $10 \mathrm{~m}$. Each tank is $1.8 \mathrm{~m}$ in diameter, $1.3 \mathrm{~m}$ in height, and filled with transparent ice to a height of $0.9 \mathrm{~m}$. A tank contains two optical sensors, each consisting of a 10-inch Hamamatsu photomultiplier tube together with electronic boards for detection, digitization, and readout (Abbasi et al. 2009, 2010). The two sensors are operated at different gains for increased dynamic range. The IceTop trigger condition requires at least three stations to have recorded hits within a $5 \mu$ s time window (Abbasi et al. 2013). IceTop detects showers at a rate of approximately $30 \mathrm{~Hz}$ with a minimum primary particle energy threshold of about $400 \mathrm{TeV}$. Its surface location near the shower maximum makes it sensitive to the full electromagnetic component of the shower in addition to the muonic component.

Cosmic-ray reconstruction relies on the optical detection of Cerenkov radiation within tanks of ice emitted by secondary particles produced by cosmic-ray interactions in the upper atmosphere. Information from individual tanks, including position, deposited charge, and pulse timing, is used to infer the air-shower direction, core location, and shower size estimate $S_{125}$, which is related to the cosmic-ray primary energy (Aartsen et al. 2013a).

Snow accumulates on the top of stations with time, attenuating the electromagnetic portion of the shower, lowering $S_{125}$. This accumulation occurs in a nonuniform way due to wind patterns around nearby structures. Snow depth measurements for each tank are performed twice a year, allowing for depth interpolation at the time of an event. An exponential correction factor is applied during event reconstruction to the signal of each tank such that the corrected tank signal $S_{125}=S_{125}^{\text {snow }} \exp \left(x / \lambda_{\text {eff }}\right)$. Here, $S_{125}^{\text {snow }}$ is the detected signal in the tank, $x$ is the slant depth through the snow above the tank, and $\lambda_{\text {eff }}$ is the effective attentuation length due to the snow. Values for $\lambda_{\text {eff }}$ are selected such that the resulting $S_{125}$ distributions for each year are consistent. The attenuation length changes over time as the snow depth generally increases across the entire array (Rawlins et al. 2015a).

\section{RECONSTRUCTION METHODS AND DATA SET}

This analysis uses 4 yr of IceTop experimental data collected between 2010 May and 2014 May. For the first year of data (IC79), 73 stations were deployed; for each of the remaining $3 \mathrm{yr}$ (IC86), IceTop operated in its final 81-station configuration.

Event reconstructions are performed using the standard IceTop reconstruction method (Abbasi et al. 2013). The values for the snow attenuation length $\lambda_{\text {eff }}$ differ for each year and are 
listed in Table 1. The shower core location on the ground is determined by a signal-weighted likelihood fit to the shower front, with a typical resolution better than $10 \mathrm{~m}$ at the highest energies. The primary arrival direction is determined from a fit to the arrival time distributions of signals in the tanks. The angular resolution is the space angle that includes $68 \%$ of reconstructed events that would arrive from a fixed direction. This value varies between 0.2 and 0.8 depending on energy and primary mass (Rawlins et al. 2015b). Above $10 \mathrm{PeV}$, the typical angular resolution, defined as the angle from the true event direction that contains $68 \%$ of reconstructed event directions, is better than 0.5 , which is taken as the representative value in the analysis.

The shower size estimate $S_{125}$ is determined by fitting the tank signals for the expected signal at $125 \mathrm{~m}$ from the shower core location. The relationship between $S_{125}$ and primary cosmic-ray energy is determined by comparison with Monte Carlo simulations for zenith angles less than $37^{\circ}$ (Rawlins et al. 2015b). The energy resolution above $2 \mathrm{PeV}$ is better than 0.1 in $\log _{10}$ of the energy (Abbasi et al. 2013).

Events are selected by requiring a good fit to the shower lateral distribution, a reconstructed core location lying within $400 \mathrm{~m}$ of the array center (not near the array boundary), and a cut on zenith angle within $37^{\circ}$. Requiring the reconstructed cores within $400 \mathrm{~m}$ yields a fiducial area $A=5.02 \times 10^{5} \mathrm{~m}^{2}$. For the final event selection for the all-sky search, we select energies above $10 \mathrm{PeV}$, and $100 \mathrm{PeV}$ for the targeted search, resulting in 1,233,487 and 12,558 events, respectively. The total live time is 1363.8 days. Table 1 lists the live time, number of events for each energy threshold, and effective snow attenuation length for each year.

The targeted search uses a higher-energy cut since most astrophysical objects of interest for this search lie at Galactic distances of order $1 \mathrm{kpc}$ or greater. This cut is also motivated by the fact the lower-energy neutrons will not typically survive from $1 \mathrm{kpc}$ and that lower energy contains only background contributions.

\section{SEARCH METHODS}

For both search methods, top-hat search windows are drawn on the sky. This procedure allows for selecting events using a hard cut on the space angle between the event direction and the window center. The locations of these search windows are described in the following sections with more detailed information about the two searches. The radius of the search window in both searches is based on the actual IceTop pointspread function and is chosen such that it optimizes the sensitivity to a point source. Point-source sensitivity is optimized by choosing a window size $\chi$ based on the angular resolution. The point-spread function is taken to be $p(\theta)=\left(\theta / \sigma^{2}\right) \exp \left(-\theta^{2} / 2 \sigma^{2}\right)$, where $\sigma=\psi / 1.51$. Here, $\theta$ is the space angle between the reconstructed and true arrival directions and $\psi$ is the angular resolution. Using top-hat search windows, the sensitivity is optimized with $\chi=1.59 \sigma=$ $1.05 \psi$, or 0.52 .

To find a signal excess within a search window, one must first know the expected number of events without signal, i.e., the background expectation value. The background value for each search window is determined by time-scrambling the data set many times. Each time-scrambled set has the same number of events as the data set. For each event, we keep its zenith and azimuth angles in detector coordinates and randomly select another time in the data set within a $24 \mathrm{hr}$ window centered on the time of the event. The search window content of the background expectation map is taken as the mean content of $10^{3}$ and $10^{6}$ time-scrambled maps for the all-sky and targeted searches, respectively.

\subsection{All-sky Search}

In the all-sky search, we look for excesses within search windows located in all parts of the sky within the field of view of IceTop. These windows are centered on the pixels of a highresolution HEALPix (Górski et al. 2004) map. $N_{\text {side }}$ is a parameter used to define and generate the map's pixels, with higher values generating higher-resolution maps. We select a map defined by $N_{\text {side }}=128$, which provides 19,800 points within the IceTop field of view and simply provides central locations from which to draw the search windows. The typical spacing between adjacent window locations in this map is 0.46 . Although window overlap will cause correlations between neighboring windows, this ensures that all events are counted. The data are first binned using a HEALPix map ("bin map") with higher resolution $\left(N_{\text {side }}=256\right)$ than the search window map. The content of a given search window is the sum of contents of those pixels in the bin map whose centers fall within the search window. The summed content of a search window is labeled $n\left(n_{b}\right)$ for the data set (background).

Statistical significance of signals within search windows is based on the observed number of events $n$ and the background expectation value $n_{b}$. The significance value of a given search window is calculated using the Li-Ma method (Li \& Ma 1983) shown in Equation (1),

$$
S=\frac{n-n_{b}}{\left|n-n_{b}\right|} \sqrt{2}\left(n \ln \left(\frac{n+\alpha n}{n_{b}+\alpha n}\right)+\frac{n_{b}}{\alpha} \ln \left(\frac{n_{b}+\alpha n_{b}}{n_{b}+\alpha n}\right)\right)^{1 / 2},
$$

where we have replaced the Li-Ma parameters $N_{\text {on }}$ and $N_{\text {off }}$ with $n$ and $n_{b} / \alpha$, respectively. The Li-Ma method is used only for the all-sky search. Typically $\alpha$ is the ratio of time spent observing on-source to the time spent observing an equivalent off-source solid angle. Here, the parameter $\alpha$ is taken to be the ratio $n_{b} / \xi$, where $\xi$ is the sum of the contents of all search windows lying within $\pm 90^{\circ}$ in R.A. and \pm 0.52 in decl. of the search window of interest, excluding the content value $n_{b}$ of the search window itself. This definition of $\alpha$ provides a local estimate of $N_{\text {off }}$ for each search window. IceTop observes largescale anisotropy in cosmic-ray arrival directions for energies above roughly $1 \mathrm{PeV}$ (Aartsen et al. 2013b); for example, a large deficit in the cosmic-ray arrival direction distribution is observed from $30^{\circ}$ to $120^{\circ}$ in R.A. The estimate of $N_{\text {off }}$ should be representative of the expected cosmic-ray flux in the vicinity of the search window, so this definition for $\alpha$ eliminates bias due to averaging over the field of view.

\subsection{Targeted Search}

The targeted search is performed to look for correlations of event directions with known nearby Galactic objects. We calculate the Poisson probability $p\left(n, n_{b}\right)$ for observing $n$ or more events within the search window expecting $n_{b}$ for each object. Fisher's method (Fisher 1925) combines a set of independent probabilities to determine a single measure of 
significance $P_{F}$ for the set. For a sequence of $p$-values $p_{1}, p_{2}, \ldots, p_{n}$, their product is $\pi=\prod_{i=1}^{n} p_{i}$. Fisher's method allows us to calculate the chance probability that a product $\pi$ of $n p$-values obtained uniformly randomly would be less than or equal to the product $\pi_{\text {obs }}$ of the $n p$-values observed: $P_{F}\left(\pi \leqslant \pi_{\text {obs }}\right)$.

A supplemental measure of significance $P_{G}$ is provided by Good's method (Good et al. 1955), which allows for weights to be assigned to each probability. In a similar way to Fisher's method, for a sequence of $p$-values $p_{i}$ with weight $w_{i}$, the weighted product $\pi_{w}=\prod_{i=1}^{n} p_{i}^{w_{i}}$. Good's method allows us to calculate the chance probability that a product $\pi_{w}$ of $n p$-values obtained uniformly randomly with weights $w_{i}$ would be less than or equal to the product $\pi_{\mathrm{w}, \text { obs }}$ of the $n p$-values observed: $P_{G}\left(\pi_{w} \leqslant \pi_{\mathrm{w}, \mathrm{obs}}\right)$. Here, these weights are proportional to the object's recorded electromagnetic flux listed in the catalog, its relative exposure to IceTop, and an expected flux attenuation factor. This factor is equal to the survival probability for a neutron with energy equal to the median energy of an $E^{-2}$ energy spectrum between $100 \mathrm{PeV}$ and $1 \mathrm{EeV}$ to arrive from the distance of a candidate source object. The weights are normalized such that their sum is 1 for each target set.

Treating the unweighted and weighted probabilities $\left(P_{F}, P_{G}\right)$ as individual test statistics, we calculate the fraction of timescrambled data sets with corresponding values less than that observed with the data. This post-trials fraction is an unbiased indicator of the correlation probability between the data set and each source set. Both the weighted and unweighted probabilities and corresponding post-trials fractions are reported. The unweighted probability is independent of the assumption that neutron emission is proportional to the electromagnetic emission and of how the flux, relative exposure, and decay probability are used to construct the object weight.

\subsection{Target Catalogs}

We consider three distinct classes: millisecond pulsars (Manchester et al. 2005) (msec), $\gamma$-ray pulsars (Abdo et al. 2013) ( $\gamma$-ray), and high-mass X-ray binaries (HMXB) (Liu et al. 2007). The msec catalog ${ }^{55}$ provides a comprehensive list of rotation-powered pulsars. The $\gamma$-ray catalog is the second Fermi-LAT pulsar catalog. The HMXB catalog ${ }^{56}$ represents a comprehensive selection of X-ray sources, composed of a compact object orbiting a massive OB class star. These classes are considered candidate sources due to their independent evidence for high-energy particle production and high flux measured at Earth. The Galactic center lies outside the zenith angle cut, lies well beyond the effective neutron range even at energies of a few hundred $\mathrm{PeV}$, and is not considered a candidate in this search.

Only objects with known distances are included in the final catalog selection. Distances for each candidate are crosschecked with the TeVCat catalog. ${ }^{57}$ Most objects are eliminated from each catalog by the zenith angle cut and by requiring that the distance is known. Sources that further appear in multiple sets are retained only in the smaller set, resulting in 17 objects in the $\gamma$-ray set, 16 objects in the msec set, and 20 objects in the HMXB set as shown in Tables 2-4, respectively. The columns in each table are the object

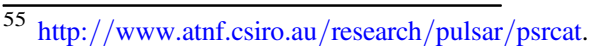

56 http://heasarc.gsfc.nasa.gov/w3browse/all/hmxbcat.html.

57 http://tevcat.uchicago.edu
}

designation, R.A., decl., distance, electromagnetic flux as recorded in the catalog, relative exposure value to IceTop, survival probability for a neutron with energy equal to the median energy of an $E^{-2}$ energy spectrum between $100 \mathrm{PeV}$ and $1 \mathrm{EeV}$, and normalized weight value. Figure 1 shows the locations of each object in equatorial coordinates. The Galactic plane is depicted by a green band to illustrate the preferential association of the $\gamma$-ray pulsar and HMXB sets with that part of the sky.

\subsection{Flux Upper Limit Calculation}

Flux upper limits are calculated for both the all-sky and targeted searches using

$$
F_{\mathrm{UL}}=1.39 s_{\mathrm{UL}} / \zeta,
$$

where $s_{\mathrm{UL}}$ is the upper limit on the number of signal events in the search window and $\zeta=T A \cos (\theta) \epsilon$ is the exposure of IceTop, where $T$ is the live time, $A \cos (\theta)$ is the projected detector area exposed to the search window, which depends on the zenith angle $\theta$, and $\epsilon$ is the reconstruction efficiency (taken as $95 \%$ according to Monte Carlo studies). The signal upper limit $s_{\mathrm{UL}}$ is calculated using a $90 \%$ Feldman-Cousins confidence level (Feldman \& Cousins 1998) based on $n$ and $n_{b}$ for the search window. The factor 1.39 is a compensation factor to include signal events that fall outside the search window. The search window includes only $71.8 \%$ of signal events based on the top-hat window and the assumed IceTop point-spread function; therefore, $s_{\mathrm{UL}}$ is scaled by $1 /$ $0.718=1.39$.

The flux upper limit can be rewritten as

$$
F_{\mathrm{UL}}=0.776\left(s_{\mathrm{UL}} / \cos (\theta)\right)\left(\mathrm{km}^{-2} \mathrm{yr}^{-1}\right),
$$

by substituting $T A \epsilon=1.79 \mathrm{~km}^{2}$ years. For an assumed $E^{-2}$ energy spectrum over the $100 \mathrm{PeV}-1 \mathrm{EeV}$ energy decade, the median energy is $181.8 \mathrm{PeV}$. The median energy flux upper limit in $\left(\mathrm{eV} \mathrm{cm}^{-2} \mathrm{~s}^{-1}\right)$ over this energy range can be written as

$$
F_{\mathrm{UL}}^{E}=0.447\left(s_{\mathrm{UL}} / \cos (\theta)\right) \text {. }
$$

Over the $10 \mathrm{PeV}-1 \mathrm{EeV}$ energy decades, the median energy is $19.80 \mathrm{PeV}$, so the conversion factor between the particle flux and median energy flux upper limits is

$$
1 \text { part. } \mathrm{km}^{-2} \mathrm{yr}^{-1}=0.0628 \mathrm{eV} \mathrm{cm}^{-2} \mathrm{~s}^{-1} \text {. }
$$

An important point to note is that Equation (4) assumes an $E^{-2}$ energy spectrum as measured at Earth, which is related to the source energy spectrum only after accounting for neutron decay factors that depend on the source distance. Figure 2 shows the attenuation factor of the energy spectrum injected at the source due to decay during propagation for representative distances. For a sufficiently distant source, the source spectrum would be harder than that observed at Earth. The lower-energy portions of the spectrum are increasingly suppressed with distance as these neutrons are removed.

The attenuation curves in Figure 2 have a strong effect on the sensitivity of the searches. The all-sky search uses a $10 \mathrm{PeV}$ energy threshold; thus, it is sensitive only to sources at extremely close distances due to the large number of events lying near threshold. The targeted search is sensitive mostly to higher neutron energies closer to $\mathrm{EeV}$ energies, which are capable of crossing larger Galactic distances. For example, a 
Table 2

Characteristics of the Fermi $\gamma$-ray Catalog

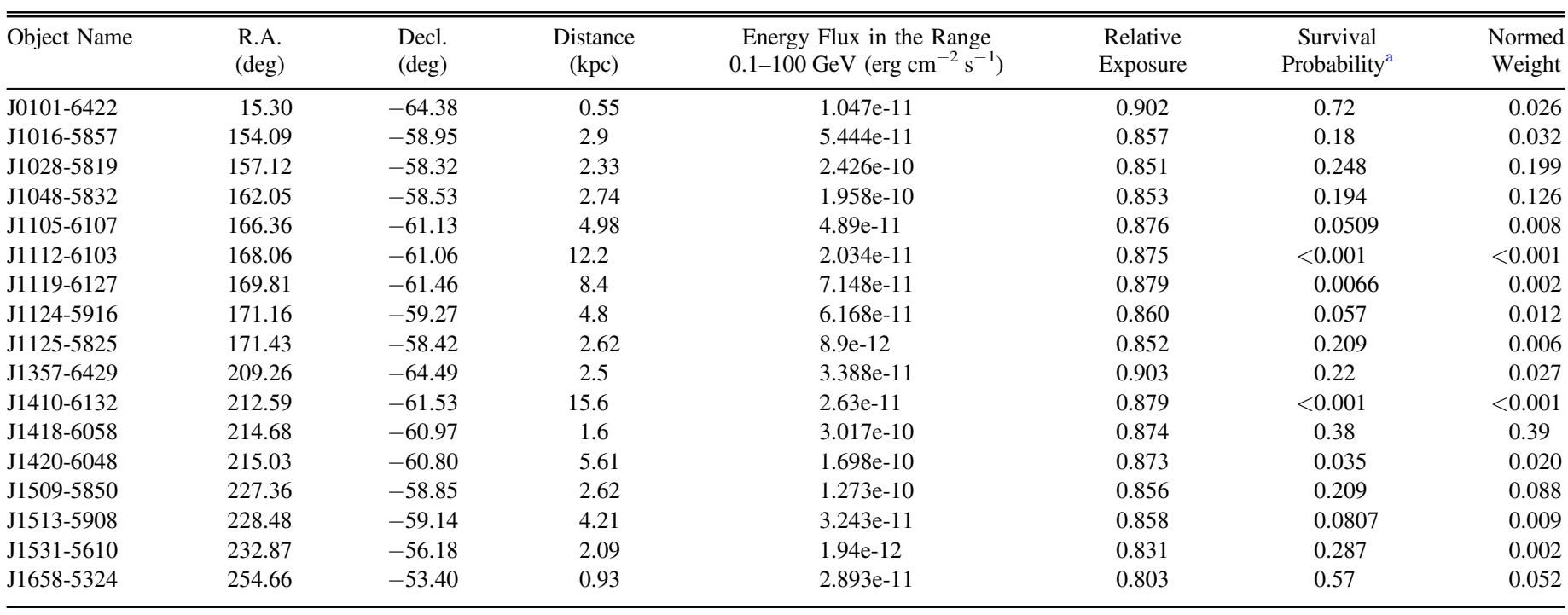

Note.

${ }^{\text {a }}$ Calculated using the median energy of an $E^{-2}$ spectrum between $100 \mathrm{PeV}$ and $1 \mathrm{EeV}$.

Table 3

Characteristics of the Msec Catalog

\begin{tabular}{|c|c|c|c|c|c|c|c|}
\hline Object Name & $\begin{array}{l}\text { R.A. } \\
\text { (deg) }\end{array}$ & $\begin{array}{l}\text { Decl. } \\
(\text { deg })\end{array}$ & $\begin{array}{l}\text { Distance } \\
\quad(\mathrm{kpc})\end{array}$ & $\begin{array}{l}\text { Energy Flux at Sun } \\
\quad\left(\mathrm{erg} \mathrm{kpc}^{-2} \mathrm{~s}^{-1}\right)\end{array}$ & $\begin{array}{l}\text { Relative } \\
\text { Exposure }\end{array}$ & $\begin{array}{c}\text { Survival } \\
\text { Probability }^{\mathrm{a}}\end{array}$ & $\begin{array}{l}\text { Normed } \\
\text { Weight }\end{array}$ \\
\hline J1017-7156 & 154.46 & -71.94 & 0.26 & $1 e+35$ & 0.951 & 0.86 & 0.5 \\
\hline B0021-72F & 6.02 & -72.08 & 4 & $8.8 \mathrm{e}+33$ & 0.951 & 0.09 & 0.005 \\
\hline J1910-5959A & 287.93 & -59.97 & 4.5 & $1.6 e+32$ & 0.866 & 0.068 & $<0.001$ \\
\hline J1103-5403 & 165.89 & -54.06 & 3.16 & $3.7 e+32$ & 0.809 & 0.151 & $<0.001$ \\
\hline J1216-6410 & 184.03 & -64.17 & 1.71 & $4.9 \mathrm{e}+32$ & 0.900 & 0.360 & 0.001 \\
\hline J2129-5721 & 322.34 & -57.35 & 0.4 & $9.9 \mathrm{e}+34$ & 0.842 & 0.8 & 0.4 \\
\hline J1431-5740 & 217.76 & -57.67 & 4.07 & $2.2 \mathrm{e}+32$ & 0.845 & 0.0877 & $<0.001$ \\
\hline J0711-6830 & 107.98 & -68.51 & 1.04 & $3.3 e+33$ & 0.931 & 0.537 & 0.010 \\
\hline J1629-6902 & 247.29 & -69.05 & 1.36 & $9.9 e+32$ & 0.934 & 0.443 & 0.003 \\
\hline J2236-5527 & 339.22 & -55.46 & 2.03 & $2.8 \mathrm{e}+32$ & 0.824 & 0.297 & $<0.001$ \\
\hline J1757-5322 & 269.31 & -53.37 & 1.36 & $8 e+32$ & 0.803 & 0.443 & 0.002 \\
\hline
\end{tabular}

Note.

${ }^{\text {a }}$ Calculated using the median energy of an $E^{-2}$ spectrum between $100 \mathrm{PeV}$ and $1 \mathrm{EeV}$.

suppression factor $S$ can be defined as the ratio between the number of neutrons with an injected $E^{-2}$ spectrum observed after including attenuation and the number observed not including attenuation for the same $E^{-2}$ spectrum. For an $E^{-2}$ spectrum between $10 \mathrm{PeV}$ and $1 \mathrm{EeV}$, removal of half the neutrons from the observed spectrum, or $S=0.5$, corresponds to a propagation distance of about $0.15 \mathrm{kpc}$. Between $100 \mathrm{PeV}$ and $1 \mathrm{EeV}, S=0.5$ corresponds to a distance of about $1.25 \mathrm{kpc}$. Generally speaking, the sensitivity of any neutron search will be shifted toward the higher-energy portion of the injected energy spectrum at the source due to decay, unless sources are sufficiently close that decay does not significantly modify the energy spectrum. This can be seen in Figure 3, which shows an example $E^{-2}$ energy spectrum modified by the distance-dependent decay attenuation.
These flux limits are time-averaged values based on the IceTop exposure $\zeta$. Particularly for the objects in the targeted source sets, it is possible that transient fluxes may temporarily exceed these limits. The energy flux limits derived from Equation (4) are strongly dependent on the assumption that an injected $E^{-2}$ energy spectrum at the source is not strongly modified in the energy range the limit applies to by neutron decay en route.

\section{RESULTS}

\subsection{All-sky Search}

Figures 4 and 5 show the differential and cumulative distributions of the $19,800 \mathrm{Li}-\mathrm{Ma}$ values compared to the isotropic expectation. In both figures, the blue and green lines 
Table 4

Characteristics of the HMXB Catalog

\begin{tabular}{|c|c|c|c|c|c|c|c|}
\hline Object Name & $\begin{array}{l}\text { R.A. } \\
\text { (deg) }\end{array}$ & $\begin{array}{l}\text { Decl. } \\
(\operatorname{deg})\end{array}$ & $\begin{array}{l}\text { Distance } \\
\quad(\mathrm{kpc})\end{array}$ & $\begin{array}{l}\text { Energy Flux in the Range } \\
\quad 2-10 \mathrm{keV}(\mu \mathrm{Jy})\end{array}$ & $\begin{array}{l}\text { Relative } \\
\text { Exposure }\end{array}$ & $\begin{array}{c}\text { Survival } \\
\text { Probability }^{\mathrm{a}}\end{array}$ & $\begin{array}{l}\text { Normed } \\
\text { Weight }\end{array}$ \\
\hline $1 \mathrm{H} \mathrm{0739-529}$ & 116.85 & -53.33 & 0.52 & 0.7 & 0.802 & 0.73 & 0.007 \\
\hline 1H 0749-600 & 117.57 & -61.10 & 0.4 & 0.7 & 0.875 & 0.8 & 0.008 \\
\hline GRO J1008-57 & 152.44 & -58.29 & 5 & 1200 & 0.851 & 0.05 & 0.9 \\
\hline RX J1037.5-5647 & 159.40 & -56.80 & 5 & 3.3 & 0.837 & 0.05 & 0.002 \\
\hline 1A $1118-615$ & 170.24 & -61.92 & 5 & 0.1 & 0.882 & 0.05 & $<0.001$ \\
\hline 4U 1119-603 & 170.31 & -60.62 & 9 & 10 & 0.871 & 0.005 & $<0.001$ \\
\hline IGR J11215-5952 & 170.44 & -59.86 & 8 & 42 & 0.865 & 0.008 & 0.005 \\
\hline 2S $1145-619$ & 177.00 & -62.21 & 2.3 & 4 & 0.885 & 0.25 & 0.02 \\
\hline 1E 1145.1-6141 & 176.87 & -61.95 & 8 & 4 & 0.883 & 0.008 & $<0.001$ \\
\hline 4U 1223-624 & 186.66 & -62.77 & 3 & 9 & 0.889 & 0.2 & 0.02 \\
\hline 1H 1249-637 & 190.71 & -63.06 & 0.3 & 2.2 & 0.891 & 0.8 & 0.03 \\
\hline 1H 1253-761 & 189.81 & -75.37 & 0.24 & 0.6 & 0.968 & 0.87 & 0.008 \\
\hline $1 \mathrm{H} 1255-567$ & 193.65 & -57.17 & 0.11 & 0.8 & 0.840 & 0.94 & 0.01 \\
\hline 4U 1258-61 & 195.32 & -61.60 & 2.4 & 0.3 & 0.880 & 0.24 & 0.001 \\
\hline 2RXP J130159.6-635806 & 195.50 & -63.97 & 5.5 & 6.3 & 0.890 & 0.037 & 0.003 \\
\hline SAX J1324.4-6200 & 201.11 & -62.01 & 3.4 & 0.4 & 0.883 & 0.1 & $<0.001$ \\
\hline 2S $1417-624$ & 215.30 & -62.70 & 6 & 2 & 0.889 & 0.03 & $<0.001$ \\
\hline SAX J1452.8-5949 & 223.21 & -59.82 & 9 & 0.045 & 0.864 & 0.005 & $<0.001$ \\
\hline XTE J1543-568 & 236.00 & -56.77 & 10 & 8 & 0.836 & 0.003 & $<0.001$ \\
\hline $1 \mathrm{H} 1555-552$ & 238.59 & -55.33 & 0.96 & 1.7 & 0.822 & 0.56 & 0.013 \\
\hline
\end{tabular}

Note.

${ }^{\text {a }}$ Calculated using the median energy of an $E^{-2}$ spectrum between $100 \mathrm{PeV}$ and $1 \mathrm{EeV}$.

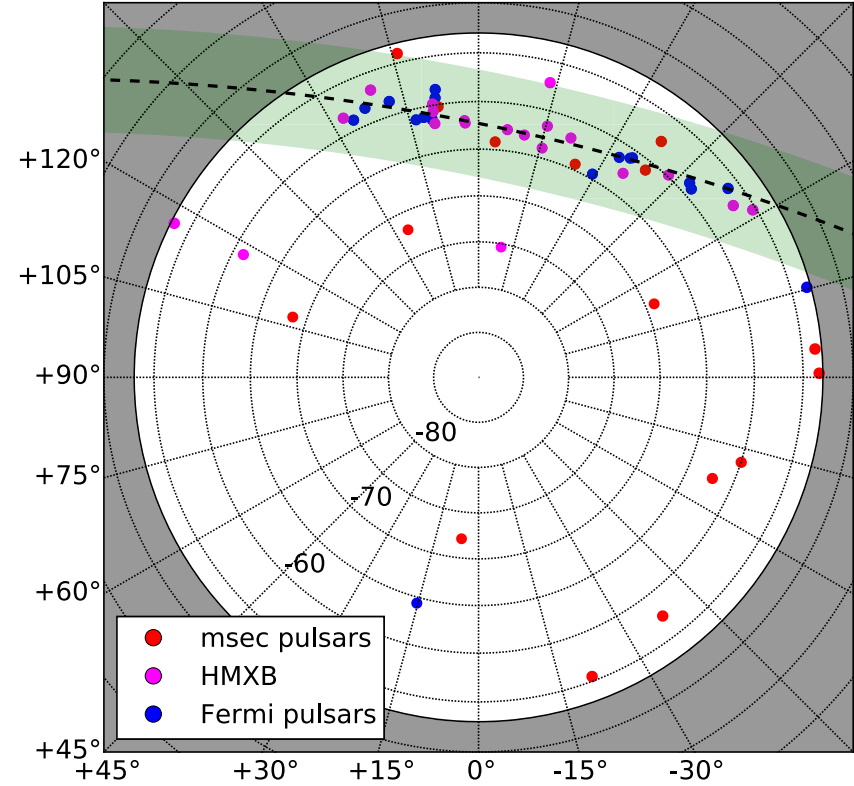

Figure 1. Equatorial polar sky map of each catalog set. The dashed black line indicates the Galactic plane, and the green band shows $b= \pm 5^{\circ}$. Each circle is 0.5 in radius.

show the Li-Ma significance distribution for the data and isotropy, respectively. There are no Li-Ma values larger than 4. The dashed line shows the Gaussian form expected for the distribution to follow if deviations from isotropy are due only to statistical fluctuations. In Figure 5, the gray shaded region in the cumulative plot shows the $95 \%$ containment band for isotropy; the presence of search windows with statistically significant signal excess would extend above and to the right of this band. The absence of such a feature indicates that no statistically significant signal excess is observed and that the

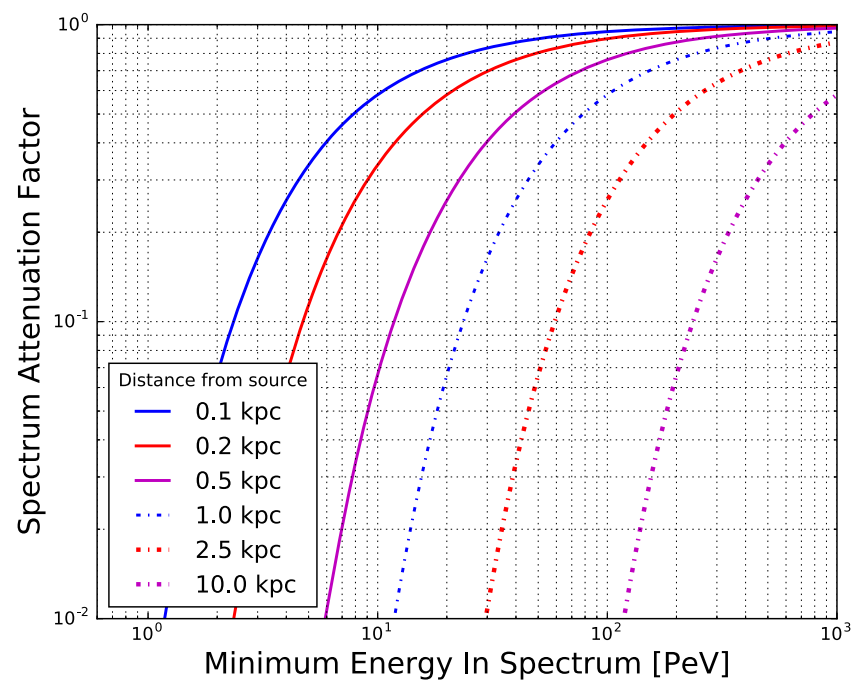

Figure 2. Spectrum attentuation factor due to neutron decay as a function of minimum energy for an $E^{-2}$ spectrum and distance from the source. The attenuation factor is a function of the median energy, which itself depends on the minimum energy.

observed excesses are consistent with fluctuations about the expectation.

Figures 6 and 7 show sky maps of the Li-Ma and flux upper limit values for each search window. No statistically significant clustering on the sky is observed, including the Galactic plane depicted by the black dashed $\left(b=0^{\circ}\right)$ and solid $\left(b= \pm 5^{\circ}\right)$ lines. As noted previously, the energies of most events used in this search lie close to the $10 \mathrm{PeV}$ energy cut, which corresponds to a neutron range of order $100 \mathrm{pc}$. The sphere from which signal could arrive is contained within the Galactic disk, so any excesses arising from cosmic-ray interactions in the disk would be distributed over the entire field of view, not concentrated within a narrow band across the sky. 


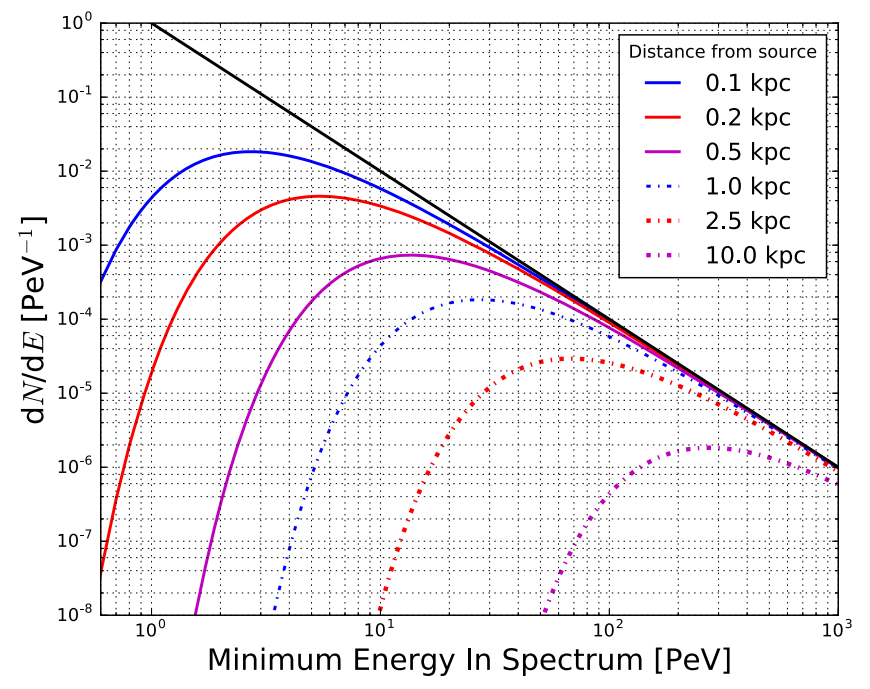

Figure 3. Effect of decay attenuation on an $E^{-2}$ energy spectrum at source as a function of minimum energy and distance from the source.

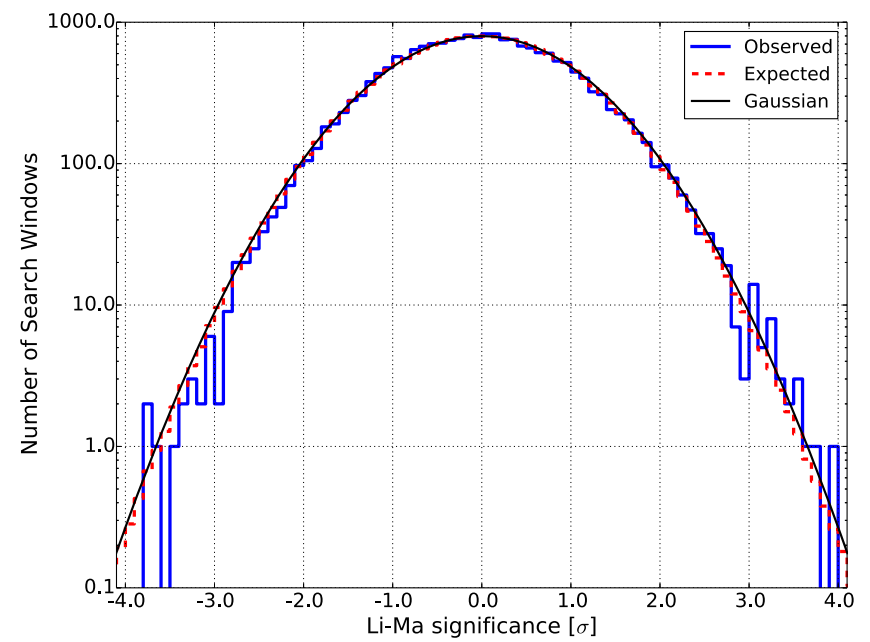

Figure 4. Differential histograms of Li-Ma values (blue) and the isotropic expectation (green). The dashed line shows the Gaussian approximation for the expected $\mathrm{Li}-\mathrm{Ma}$ distribution in the case in which deviations result only from statistical fluctuations.

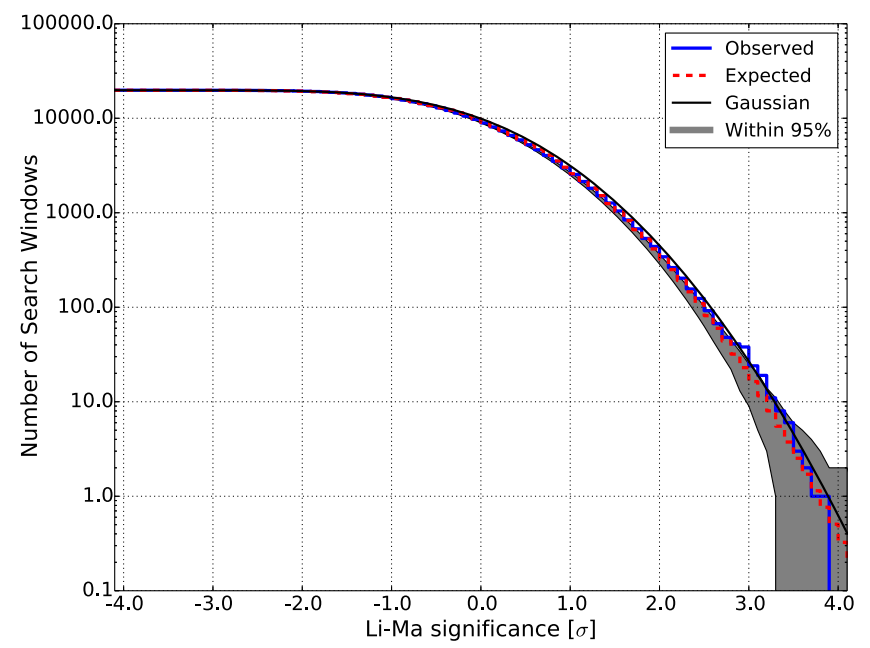

Figure 5. Cumulative histograms of Li-Ma values (blue) and the isotropic expectation (green).

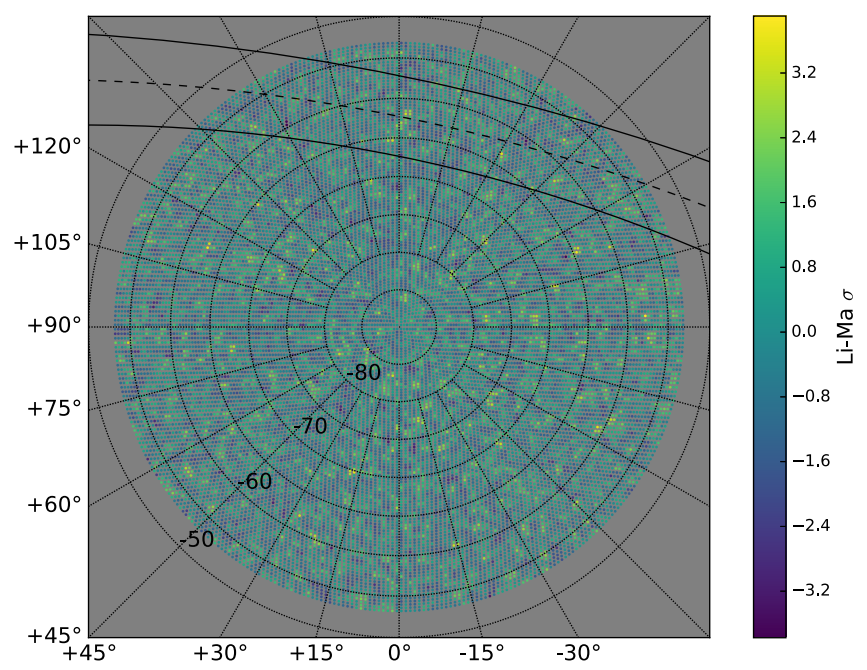

Figure 6. Equatorial polar sky map of $\mathrm{Li}-\mathrm{Ma}$ values for each search window. The dashed black line indicates the Galactic plane, and the solid black lines depict $b= \pm 5^{\circ}$.

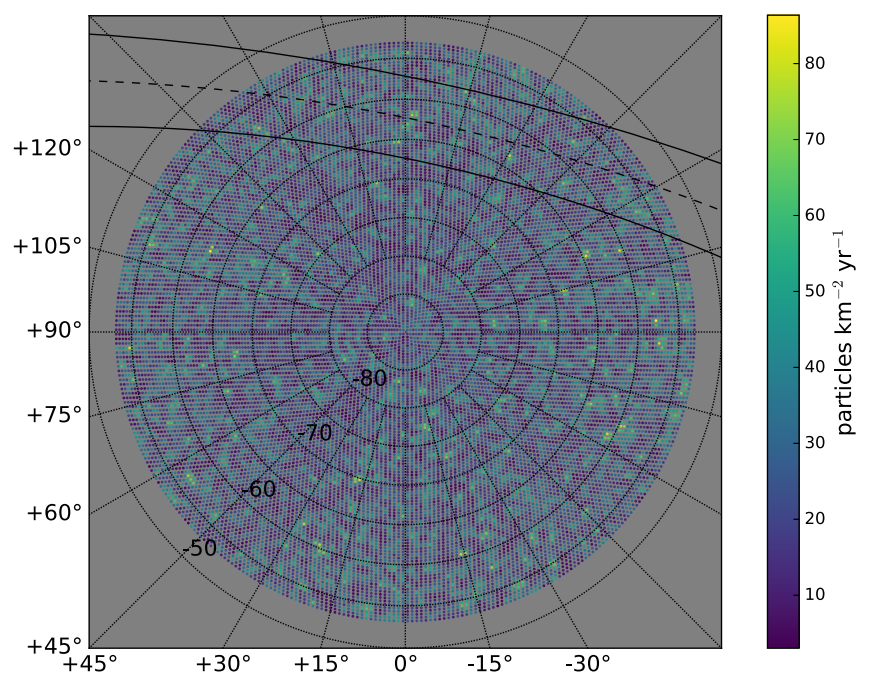

Figure 7. Equatorial polar sky map of flux upper limit values for each search window. The dashed black line indicates the Galactic plane, and the solid black lines depict $b= \pm 5^{\circ}$.

Figure 8 shows the mean flux upper limit as a function of declination for the all-sky search. The limits are strongest near the South Pole due to the maximal exposure, but there is greater uncertainty on the mean since there are fewer search windows in declination bands closest to the pole.

\subsection{Targeted Search}

Table 5 lists the correlation probabilities for each catalog, with the corresponding post-trials probability in parentheses. No significant correlation is observed with any catalog. Tables 6-8 give details of each object. The columns in each table are the object designation, observed number of events within the search window, background estimate in the window, particle flux above $100 \mathrm{PeV}$ according to Equation (3), energy flux above $100 \mathrm{PeV}$ according to Equation (4), and Poisson probability $p\left(n, n_{b}\right)$ for observing $n$ events with an expectation number $n_{b}$. These flux limits assume an $E^{-2}$ energy spectrum as measured at Earth. The most significant object in each catalog is highlighted in bold. 


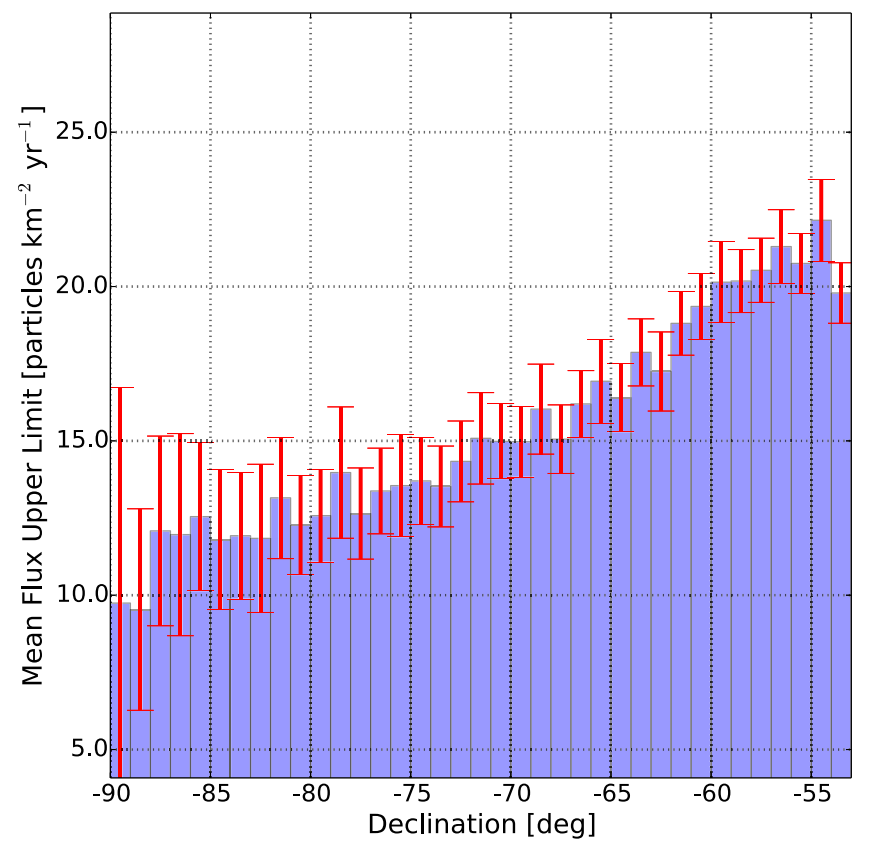

Figure 8. Mean flux upper limit $\left(90 \%\right.$ C.L.) for $1^{\circ}$ declination bins for the allsky search. The error bars indicate the statistical uncertainty on the mean value since there are many search windows within each declination band.

Table 5

Targeted Search Results with Each Catalog

\begin{tabular}{lcc}
\hline \hline Catalog & Unweighted $P_{F}$ & Weighted $P_{G}$ \\
\hline$\gamma$-ray & $0.999(0.976)$ & $0.910(0.776)$ \\
msec & $0.809(0.408)$ & $0.888(0.778)$ \\
HMXB & $0.999(0.988)$ & $0.946(0.971)$ \\
\hline
\end{tabular}

Note. Values in parentheses give the post-trials probability.

The catalog probabilities do not appear to be distributed uniformly between 0 and 1 , since at least one probability value would be expected to lie below 0.809 in over $99.5 \%$ of sets of six uniformly distributed random samples. There exists an underfluctuation in the data along $b=0^{\circ}$ compared to the background expectation, as illustrated in Figure 9. The preferential clustering of the $\gamma$-ray pulsar and HMXB catalogs along the Galactic plane, combined with this underfluctuation, acts to drive the catalog probabilities to higher values. Since typically $n<n_{b}$, the individual Poisson $p$-values are close to 1 . This is checked by rotating these catalogs by a prescribed amount in R.A. and expecting lower catalog probabilities due to higher $n$ and similar $n_{b}$ in the windows. These values are shown in Table 9 for different rotation values.

We also note that there are four pairs of objects that lie within $1^{\circ}$ of each other. In all four cases, the objects in each pair are distinct from each other, lie at different distances, and are from different catalogs. We find consistent results with Table 5 when we mask the object with the farther distance.

\section{SUMMARY AND DISCUSSION}

IceTop does not observe a statistically significant point source of cosmic-ray arrival directions. Using Equation (5), the all-sky mean flux upper limits for individual declination bands correspond to energy fluxes between about 0.6 and $1.2 \mathrm{eV} \mathrm{cm}^{-2} \mathrm{~s}^{-1}$ between $100 \mathrm{PeV}$ and $1 \mathrm{EeV}$ assuming an $E^{-2}$ neutron energy spectrum as measured at Earth, which are comparable to $\mathrm{TeV}$ photon fluxes for Galactic objects (Hinton \& Hofmann 2009). These flux limits are the first neutron flux upper limits in the southern hemisphere for energies in the $10 \mathrm{PeV}$ to $1 \mathrm{EeV}$ energy decades. Again, it is important to note that neutron decay en route will modify the energy spectrum as illustrated in Figure 2, so the source spectrum would be generally softer than that constrained. The limits in both searches are strongly dependent on the assumption that an injected $E^{-2}$ spectrum is not significantly modified by decay, as noted in Section 4.4. For the all-sky search, this restricts the applicability of the limits within a small volume around Earth. For the targeted search, there are a number of objects that lie within $1 \mathrm{kpc}$, so their limits are most compatible with the base assumption.

As noted previously, hadronic production of photons by protons with an $E^{-2}$ spectrum will inject equal power into each energy decade, and the neutron production at least equals the photon production. At present, these flux upper limits do not

Table 6

Targeted Search Results for the Fermi $\gamma$-Ray Catalog

\begin{tabular}{|c|c|c|c|c|c|}
\hline Object Name & $\begin{array}{c}\text { Observed } \\
n\end{array}$ & $\begin{array}{l}\text { Background } \\
\text { Estimate } n_{b}\end{array}$ & $\begin{array}{c}F_{\mathrm{UL}} \\
\left(\mathrm{km}^{-2} \mathrm{yr}^{-1}\right)\end{array}$ & $\begin{array}{c}F_{\mathrm{UL}}^{E} \\
\left(\mathrm{eV} \mathrm{cm} \mathrm{cm}^{-2}\right)\end{array}$ & $\begin{array}{c}\text { Poisson } \\
\text { Probability } p\end{array}$ \\
\hline J1016-5857 & 3 & 2.62 & 4.35 & 2.51 & 0.487 \\
\hline J1028-5819 & 1 & 1.80 & 2.44 & 1.41 & 0.835 \\
\hline J1048-5832 & 5 & 2.77 & 6.57 & 3.79 & 0.147 \\
\hline J1105-6107 & 2 & 3.79 & 2.19 & 1.26 & 0.892 \\
\hline J1112-6103 & 3 & 3.79 & 3.29 & 1.90 & 0.729 \\
\hline $\mathrm{J} 1125-5825$ & 2 & 2.65 & 3.02 & 1.74 & 0.742 \\
\hline J1124-5916 & 2 & 1.73 & 3.78 & 2.18 & 0.517 \\
\hline J1119-6127 & 3 & 2.71 & 4.16 & 2.40 & 0.508 \\
\hline J0101-6422 & 3 & 2.81 & 3.98 & 2.29 & 0.534 \\
\hline J1357-6429 & 2 & 2.34 & 3.09 & 1.78 & 0.679 \\
\hline J1410-6132 & 1 & 2.75 & 1.79 & 1.03 & 0.936 \\
\hline J1418-6058 & 2 & 2.81 & 2.83 & 1.63 & 0.770 \\
\hline J1420-6048 & 2 & 2.62 & 2.98 & 1.72 & 0.737 \\
\hline J1509-5850 & 0 & 2.37 & 0.83 & 0.48 & 1.000 \\
\hline J1513-5908 & 0 & 1.81 & 1.07 & 0.62 & 1.000 \\
\hline J1531-5610 & 0 & 2.78 & 0.68 & 0.39 & 1.000 \\
\hline J1658-5324 & 1 & 2.59 & 2.06 & 1.19 & 0.925 \\
\hline
\end{tabular}


Table 7

Targeted Search Results for the Msec Pulsar Catalog

\begin{tabular}{|c|c|c|c|c|c|}
\hline Object Name & $\begin{array}{c}\text { Observed } \\
n\end{array}$ & $\begin{array}{l}\text { Background } \\
\text { Estimate } n_{b}\end{array}$ & $\begin{array}{c}F_{\mathrm{UL}} \\
\left(\mathrm{km}^{-2} \mathrm{yr}^{-1}\right)\end{array}$ & $\begin{array}{c}F_{\mathrm{UL}}^{E} \\
\left(\mathrm{eV} \mathrm{cm}^{-2} \mathrm{~s}^{-1}\right)\end{array}$ & $\begin{array}{c}\text { Poisson } \\
\text { Probability } p\end{array}$ \\
\hline J0711-6830 & 2 & 2.54 & 2.85 & 1.64 & 0.720 \\
\hline J1103-5403 & 2 & 1.99 & 3.77 & 2.17 & 0.591 \\
\hline J1125-6014 & 2 & 1.80 & 3.68 & 2.12 & 0.537 \\
\hline J1216-6410 & 3 & 2.67 & 4.10 & 2.36 & 0.499 \\
\hline B $0021-72 F$ & 4 & 1.95 & 5.42 & 3.12 & 0.133 \\
\hline $\mathrm{J} 1431-5740$ & 3 & 1.84 & 5.13 & 2.96 & 0.281 \\
\hline J1629-6902 & 0 & 2.89 & 0.57 & 0.33 & 1.000 \\
\hline J2236-5527 & 4 & 2.72 & 5.54 & 3.19 & 0.289 \\
\hline J1933-6211 & 6 & 3.20 & 7.26 & 4.18 & 0.106 \\
\hline J1910-5959A & 2 & 3.21 & 2.58 & 1.49 & 0.830 \\
\hline J2129-5721 & 1 & 2.48 & 2.04 & 1.18 & 0.916 \\
\hline
\end{tabular}

Table 8

Targeted Search Results for the HMXB Catalog

\begin{tabular}{|c|c|c|c|c|c|}
\hline Object Name & $\begin{array}{c}\text { Observed } \\
n\end{array}$ & $\begin{array}{l}\text { Background } \\
\text { Estimate } n_{b}\end{array}$ & $\begin{array}{c}F_{\mathrm{UL}} \\
\left(\mathrm{km}^{-2} \mathrm{yr}^{-1}\right)\end{array}$ & $\begin{array}{c}F_{\mathrm{UL}}^{E} \\
\left(\mathrm{eV} \mathrm{cm}^{-2} \mathrm{~s}^{-1}\right)\end{array}$ & $\begin{array}{c}\text { Poisson } \\
\text { Probability } p\end{array}$ \\
\hline 1H 0739-529 & 0 & 2.51 & 0.82 & 0.47 & 1.000 \\
\hline 1H 0749-600 & 1 & 2.44 & 1.98 & 1.14 & 0.913 \\
\hline GRO J1008-57 & 1 & 2.82 & 1.82 & 1.05 & 0.941 \\
\hline RX J1037.5-5647 & 2 & 3.22 & 2.66 & 1.53 & 0.832 \\
\hline IGR J11215-5952 & 2 & 1.95 & 3.56 & 2.05 & 0.579 \\
\hline 4U 1119-603 & 2 & 1.83 & 3.63 & 2.09 & 0.546 \\
\hline 1A $1118-615$ & 1 & 2.08 & 2.18 & 1.26 & 0.876 \\
\hline 1E $1145.1-6141$ & 3 & 2.64 & 4.21 & 2.43 & 0.492 \\
\hline 2S $1145-619$ & 3 & 2.31 & 4.49 & 2.59 & 0.408 \\
\hline $4 \mathrm{U} 1223-624$ & 1 & 3.39 & 1.45 & 0.84 & 0.966 \\
\hline 1H $1249-637$ & 1 & 2.09 & 2.14 & 1.23 & 0.877 \\
\hline 1H $1253-761$ & 2 & 3.41 & 2.19 & 1.26 & 0.855 \\
\hline 1H $1255-567$ & 2 & 2.01 & 3.61 & 2.08 & 0.598 \\
\hline 2RXP J130159.6-635806 & 2 & 2.87 & 2.71 & 1.56 & 0.781 \\
\hline $4 U$ 1258-61 & 1 & 2.93 & 2.28 & 1.31 & 0.947 \\
\hline SAX J1324.4-6200 & 1 & 2.49 & 1.93 & 1.11 & 0.917 \\
\hline $2 S 1417-624$ & 4 & 2.86 & 5.01 & 2.89 & 0.322 \\
\hline SAX J1452.8-5949 & 3 & 1.69 & 5.15 & 2.97 & 0.239 \\
\hline XTE J1543-568 & 3 & 2.81 & 4.29 & 2.47 & 0.532 \\
\hline 1H $1555-552$ & 0 & 2.42 & 8.35 & 4.81 & 1.000 \\
\hline
\end{tabular}

strongly constrain the $\mathrm{TeV}$ photon production mechanism, or the shape of the parent energy spectrum. No significant correlation is found with known nearby Galactic objects characterized by $\mathrm{GeV}-\mathrm{TeV}$ energy photon emission and plausibly capable of producing PeV neutrons.

The nonobservation of $\mathrm{PeV}$ neutrons may simply indicate that these objects are not producing neutrons at these energies, or that typical Galactic neutron sources are not near Earth. Local PeV neutron production in the Galaxy could simply be episodic or transient, for example, occurring during supernova explosions or other extremely high energy particle production events. Alternatively, the sources may emit particle jets continuously, but their number may be few and the jets are not oriented toward Earth. Individual sources could emit weakly but be densely distributed.

Additionally, the environment around any sources may not be sufficiently dense to facilitate neutron production by cosmic- ray interaction such that the primaries escape the acceleration region into interstellar space before interacting and producing neutrons. In this case, neutrons decay in interstellar space relatively near the primary source producing secondary protons (Bednarek \& Protheroe 1997). These secondary protons then propagate diffusively in the GMF, so sources that are sufficiently far away will not manifest a point-source signal of cosmic-ray neutrons, but could contribute to a proton signal that is smeared on the sky and not necessarily pointing back to the original source; this argument was presented by Bossa et al. (2003) when they considered EeV neutrons from the Galactic center. At PeV energies, neutrons would penetrate much less into the surrounding medium, so any potential signal from the resulting protons would be strongly suppressed by the scattering effects of the GMF and masked by the background cosmic-ray flux. 

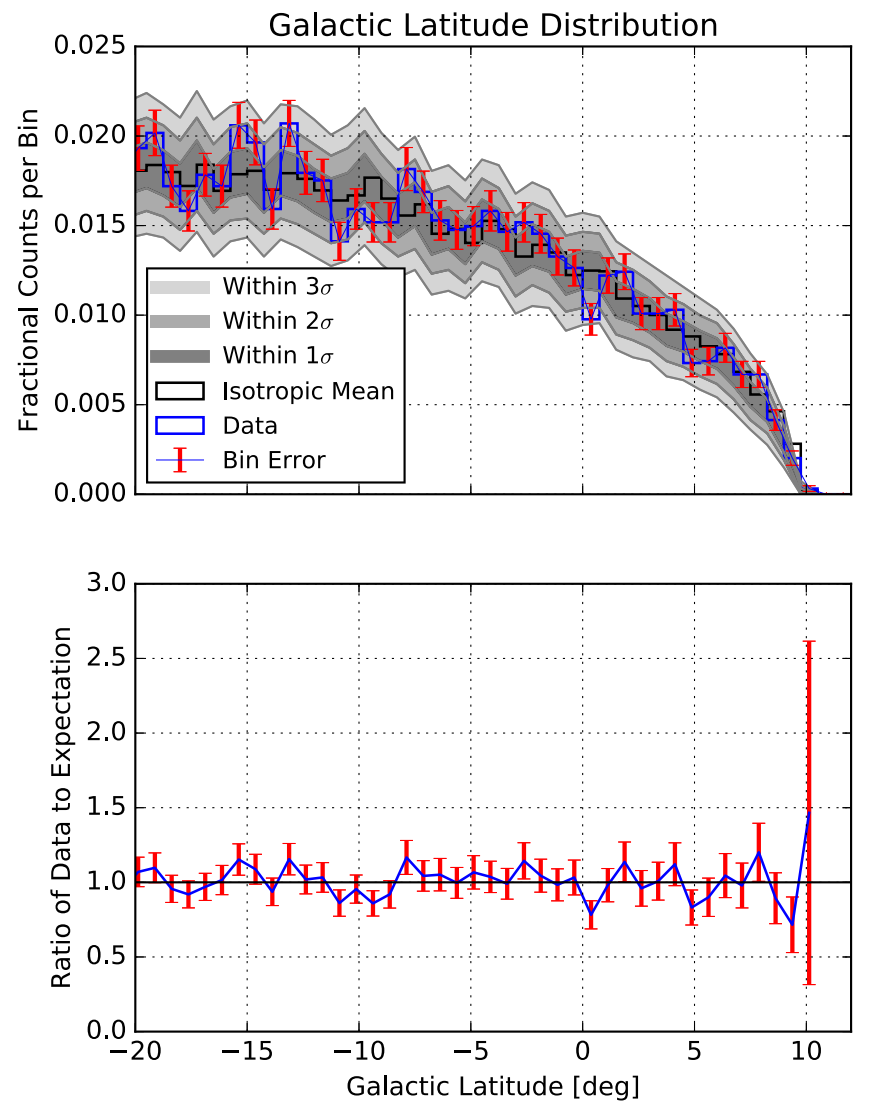

Figure 9. Distribution of number of events above $100 \mathrm{PeV}$ as a function of Galactic latitude. In the top frame, the blue histogram shows the data; the black line shows the isotropic expectation from 10,000 time-scrambled data sets. The red error bars show the Poisson uncertainty in the data histogram. The gray shaded bands depict the $68 \%, 95 \%$, and $99 \%$ containment bands for isotropy in each latitude bin. The bottom frame shows the ratio between the data and isotropic expectation.

Table 9

Results of R.A. Rotation Tests for $\gamma$-Ray Pulsar and HMXB Catalogs

\begin{tabular}{lccc}
\hline \hline Probability & $+45^{\circ}$ & $+90^{\circ}$ & $+180^{\circ}$ \\
\hline HMXB $P_{F}$ & $0.070(0.002)$ & $0.991(0.828)$ & $0.978(0.741)$ \\
HMXB $P_{G}$ & $0.262(0.129)$ & $0.164(0.116)$ & $0.946(0.982)$ \\
$\gamma$-ray $P_{F}$ & $0.803(0.408)$ & $0.126(0.016)$ & $0.991(0.866)$ \\
$\gamma$-ray $P_{G}$ & $0.074(0.025)$ & $0.586(0.311)$ & $0.845(0.645)$ \\
\hline
\end{tabular}

Note. Values in parentheses give the post-trials probability.

At higher energies, for example, between 10 and $100 \mathrm{PeV}$, this process could further enrich the cosmic-ray proton fraction above that which is directly accelerated at the source. The knee in the cosmic-ray spectrum is observed around $4 \mathrm{PeV}$, which is interpreted as an indication of a maximum attainable rigidity of typical Galactic cosmic-ray sources and of associated changes in elemental composition (see, e.g., Hörandel 2005; Blasi 2014). It is plausible that the maximum attainable energy for the proton energy spectrum in nearby sources may not extend well above the knee energy, although for heavier compositions this scales with the nuclear charge $Z$. Above $10 \mathrm{PeV}$, the cosmic-ray flux becomes progressively heavier with energy and with a decreasing proton fraction, which is roughly $20 \%$ at $10 \mathrm{PeV}$ (Apel et al. 2013; Rawlins et al. 2015b). This suggests that such secondary enrichment may be unlikely since a recovering proton fraction is not observed at energies between roughly 10 and a few hundred PeV.

The nonobservation of a $\mathrm{PeV}$ neutron flux does not necessarily preclude the existence of a $\mathrm{PeV}$ photon flux. The neutron energy spectrum at lower energies becomes increasingly modified by decay. PeV photons, on the other hand, have an absorption length considerably larger than the neutron decay distance and will maintain an unmodified energy spectrum that more resembles the injected spectrum at the source. $\mathrm{PeV}$ photons could still plausibly be produced by nonhadronic processes, such as inverse-Compton scattering from a high-energy electron population in or near Galactic sources (see, e.g., Schlickeiser 1989; Balbo et al. 2011; Nozawa et al. 2011; Kohri et al. 2012), although there are flux upper limits in the northern (Chantell et al. 1997; Borione et al. 1998; Feng et al. 2015b; Kang et al. 2015a, 2015b) and southern (Aartsen et al. 2013c) hemispheres. These photon limits, except for (Kang et al. 2015a), are for energies of order $1 \mathrm{PeV}$ or below, whereas this analysis is most sensitive at energies above $100 \mathrm{PeV}$.

We acknowledge the support from the following agencies: US National Science Foundation-Office of Polar Programs, US National Science Foundation-Physics Division, University of Wisconsin Alumni Research Foundation, the Grid Laboratory Of Wisconsin (GLOW) grid infrastructure at the University of Wisconsin-Madison, the Open Science Grid (OSG) grid infrastructure; US Department of Energy, and National Energy Research Scientific Computing Center, the Louisiana Optical Network Initiative (LONI) grid computing resources; Natural Sciences and Engineering Research Council of Canada, WestGrid, and Compute/Calcul Canada; Swedish Research Council, Swedish Polar Research Secretariat, Swedish National Infrastructure for Computing (SNIC), and Knut and Alice Wallenberg Foundation, Sweden; German Ministry for Education and Research (BMBF), Deutsche Forschungsgemeinschaft (DFG), Helmholtz Alliance for Astroparticle Physics (HAP), Research Department of Plasmas with Complex Interactions (Bochum), Germany; Fund for Scientific Research (FNRSFWO), FWO Odysseus program, Flanders Institute to encourage scientific and technological research in industry (IWT), Belgian Federal Science Policy Office (Belspo); University of Oxford, United Kingdom; Marsden Fund, New Zealand; Australian Research Council; Japan Society for Promotion of Science (JSPS); the Swiss National Science Foundation (SNSF), Switzerland; National Research Foundation of Korea (NRF); Villum Fonden, Danish National Research Foundation (DNRF), Denmark.

\section{REFERENCES}

Abreu, P., Aglietta, M., Ahlers, M., et al. 2012, ApJ, 760, 148 Aab, A., Abreu, P., Aglietta, M., et al. 2014, ApJL, 789, L34 Aab, A., Abreu, P., Aglietta, M., et al. 2015, ApJ, 804, 15

Aartsen, M. G., Abbasi, R., Abdou, Y., et al. 2013a, PhRvD, 88, 042004 Aartsen, M. G., Abbasi, R., Abdou, Y., et al. 2013b, ApJ, 765, 55

Aartsen, M. G., Abbasi, R., Abdou, Y., et al. 2013c, PhRvD, 87, 062002 Abbasi, R., Abdou, Y., Abu-Zayyad, T., et al. 2010, Nucl. Instr. Meth., A, 618,139

Abbasi, R., Abdou, Y., Ackermann, M., et al. 2013, Nucl. Instr. Meth., A, 700,188

Abbasi, R., Abe, M., Abu-Zayyad, T., et al. 2015, ApJ, 804, 133

Abbasi, R., Ackermann, M., Adams, J., et al. 2009, Nucl. Instr. Meth., A, 601,294

Abdo, A., Ajello, M., Allafort, A., et al. 2013, ApJS, 208, 17

Anchordoqui, L., Beacom, J., Goldberg, H., Palomares-Ruiz, S., \& Weiler, T. 2007, PhRvD, 75, 063001 
Antoni, T., Apel, W., Badea, A., et al. 2004, ApJ, 608, 865

Apel, W., Arteaga-Velazquez, J., Bekk, K., et al. 2013, APh, 47, 54

Balbo, M., Walter, R., Ferrigno, C., \& Bordas, P. 2011, A\&A, 527, L4

Bednarek, W., \& Protheroe, R. 1997, PhRvL, 79, 2616

Bellido, J., Clay, R., Dawson, B., \& Johnston-Hollitt, M. 2001, APh, 15,167

Blasi, P. 2014, CRPhy, 15, 329

Borione, A., Catanese, M., Chantell, M., Covault, C., \& Cronin, J. 1998, ApJ, 493, 175

Bossa, M., Mollerach, S., \& Roulet, E. 2003, J. Phys. G: Nucl. Part. Phys., 29, 1409

Candia, J., Epele, L., \& Roulet, E. 2002, APh, 17, 23

Cavasinni, V., Grasso, D., \& Maccione, L. 2006, APh, 26, 41

Chantell, M., Covault, C., Cronin, J., et al. 1997, PhRvL, 79, 1805

Crocker, R., Fatuzzo, M., Jokipii, J., Melia, F., \& Volkas, R. 2005, ApJ, 622,892

Feldman, G., \& Cousins, R. 1998, PhRvD, 57, 3873

Feng, Z., Apel, W., Arteaga-Velaquez, J., et al. 2015, Proc. ICRC, 823

Fisher 1925, Statistical Methods for Research Workers (Edinburgh: Oliver and Boyd)
Good, I. J. 1955, Journ. Royal Stat. Soc. B, 17, 264

Górski, K., Hivon, E., Banday, A., et al. 2004, ApJ, 622, 759

Hayashida, N., Nagano, M., Nishikawa, D., Ohoka, H., \& Sakaki, N. 1999, $\mathrm{APh}, 10,303$

Hinton, J., \& Hofmann, W. 2009, ARA\&A, 47, 523

Hörandel, J. 2005, IJMPA, 20, 6753

Kang, D., Apel, W., Arteaga-Velazquez, J., et al. 2015a, Proc. ICRC, 810

Kang, D., Feng, Z., Apel, W., et al. 2015b, Proc. ICRC, 812

Kohri, K., Ohira, Y., Ioka, K., et al. 2012, MNRAS, 424, 2249

Li, T.-P., \& Ma, Y.-Q. 1983, ApJ, 272, 317

Liu, Q., van Paradijs, J., \& van den Heuvel, E. 2007, A\&A, 455, 1156

Manchester, R., Hobbs, G., Teoh, A., \& Hobbs, M. 2005, ApJ, 129, 1993

Nozawa, S., Kohyama, Y., Itoh, N., et al. 2011, (arXiv:1103.4284)

Olive, K. A., et al. Particle Data Group 2014, ChPhC, 38, 090001 and 2015 update

Rawlins, K., Aartsen, M., Abraham, K., et al. 2015a, Proc. ICRC, 628 (arXiv:1510.5225)

Rawlins, K., Aartsen, M., Abraham, K., et al. 2015b, Proc. ICRC, 334 (arXiv:1510.5225)

Schlickeiser, R. 1989, A\&A, 213, L23 